IJMMS 27:1 (2001) 39-44

PII. S0161171201005075

http://ijmms.hindawi.com

(C) Hindawi Publishing Corp.

\title{
A NOTE ON NONFRAGMENTABILITY OF BANACH SPACES
}

\section{S. ALIREZA KAMEL MIRMOSTAFAEE}

(Received 15 October 1999 and in revised form 4 May 2000)

\begin{abstract}
We use Kenderov-Moors characterization of fragmentability to show that if a compact Hausdorff space $X$ with the tree-completeness property contains a disjoint sequences of clopen sets, then $(C(X)$, weak) is not fragmented by any metric which is stronger than weak topology. In particular, $C(X)$ does not admit any equivalent locally uniformly convex renorming.
\end{abstract}

2000 Mathematics Subject Classification. 46B20, 46B26, 46E15, 54C45.

1. Introduction. Let $(X, \tau)$ be a topological space and $\rho$ a metric on $X$. Given $\epsilon>0$, a nonempty subset $A$ of $X$ is said to be fragmented by $\rho$ down to $\epsilon$ if each nonempty subset of $A$ has a nonempty $\tau$-relatively open subset of $A$ with $\rho$-diameter less than $\epsilon$. The set $A$ is said to be fragmented by $\rho$ if $A$ is fragmented by $\rho$ down to $\epsilon$ for each $\epsilon>0$. The set $A$ is said to be sigma-fragmented by $\rho$ [7] if for each $\epsilon>0, A$ can be expressed as $A=\bigcup_{n=1}^{\infty} A_{n, \epsilon}$ with each $A_{n, \epsilon}$ fragmented by $\rho$ down to $\epsilon$.

The notion of fragmentability was originally introduced in [11] as an abstraction of phenomena often encountered, for example, in Banach spaces with the RadonNikodym property, in weakly compact subsets of Banach spaces and in the dual of Banach spaces. The notion of $\sigma$-fragmentability appeared in [10] in order to extend the study of compact fragmented space to noncompact spaces. It turns out that the question of whether a given Banach space with weak topology is sigma-fragmented by the norm is closely connected with the question of the existence of an equivalent Kadec and locally uniformly convex norm. The reader may refer to $[6,7,8,9,10$, $11,12,13,14,15,16,17,18,19,20]$ for some application of fragmentability and its variants in other topics of Banach spaces.

Kenderov and Moors $[13,14]$ used the following topological game to characterize fragmentability and sigma-fragmentability of a topological space $X$.

Two players $\Sigma$ and $\Omega$ alternatively select subsets of $X$. The player $\Sigma$ usually starts the game by choosing some nonempty subset $A_{1}$ of $X$, then the $\Omega$-player chooses some nonempty relatively open subset $A_{1}$, say $B_{1}$, then $\Sigma$ will choose a nonempty set $A_{2} \subset B_{1}$ and in turn, $\Omega$ picks up some nonempty relatively open subset $B_{2}$ of $A_{2}$. By continuing this procedure, the two players generate a sequence of sets

$$
A_{1} \supset B_{1} \supset \cdots \supset A_{n} \supset B_{n} \supset \cdots
$$

which is called a play and is denoted by $p=\left(A_{i}, B_{i}\right)_{i=1}^{\infty}$. If

$$
p_{k}=\left(A_{1}, B_{1}, \ldots, A_{k}\right) \quad(1 \leq k \leq n)
$$

are the first " $n$ " move of some play (of the game), then we call $p_{k}$ a partial play of 
the game. The player $\Omega$ is said to have won the play if $\bigcap_{i=1}^{\infty} A_{i}$ contains at most one point. Otherwise the player $\Sigma$ is said to have won this play. Under the term strategy for the player $\Omega$, we understand a mapping $\omega$ which assigns to every partial play $p_{n}$ a nonempty relatively open subset $B_{n}=\omega\left(p_{n}\right)$ of $A_{n}$. The play $\left(A_{i}, B_{i}\right)_{i=1}^{\infty}$ is called an $\omega$-play if $B_{i}=\omega\left(p_{i}\right)$ for every $i \geq 1$. Similarly, the partial play $p_{n}$ is called a partial $\omega$-play, if $B_{i}=\omega\left(p_{i}\right)$ for each $i<n$. The map $\omega$ is called a winning strategy for the player $\Omega$ if he/she wins every $\omega$-play. If the space $X$ is fragmentable by a metric $d(\cdot, \cdot)$, then $\Omega$ has an obvious winning strategy $\omega$. Indeed, to each partial play $p_{n}$ this strategy puts into correspondence some nonempty subset $B_{n} \subset A_{n}$ which is relatively open in $A_{n}$ and has $d$-diameter less than $1 / n$. Clearly, the set $\bigcap_{i \geq 1} A_{i}=\bigcap_{i \geq 1} B_{i}$ has at most one point because it has $d$-diameter 0 . It turns out that the existence of a winning strategy for player $\Omega$ characterizes fragmentability.

THEOREM 1.1 (see [13]). The topological space $X$ is fragmentable if and only if the player $\Omega$ has a winning strategy.

By Theorem 1.1, it was shown in [15] that $X / c_{0}$, where $X$ is the Haydon-Zizler subspace of $\ell^{\infty}$ [5] is not fragmented by any metric. According to a result of Ribarska [18], if a Banach space admits an equivalent strictly convex renorming, then it is fragmented by a metric. It follows that $X / c_{0}$ does not admit strictly convex renorming. This could be considered as an extension of [1].

Although $\ell^{\infty}$ taken with its weak topology is not sigma-fragmented by the norm, it is fragmented by a lower semi-continuous metric (see [9, Example 3.2]). However, in [14], it is shown that fragmentability and sigma-fragmentability in a Banach space may be related to each other in the following way.

THEOREM 1.2 (see [14, Theorems 1.3, 1.4, and 2.1]). For a Banach space $X$ the following are equivalent:

(i) (X, weak) is sigma-fragmented by a metric which is stronger than the weak topology;

(ii) (X, weak) is fragmented by a metric which is stronger than the weak topology;

(iii) there exists a strategy $\omega$ for the player $\Omega$ such that, for every $\omega$-play $p=$ $\left(A_{i}, B_{i}\right)_{i}$ either $\bigcap_{i \geq 1} B_{i}=\varnothing$ or $\lim _{i \rightarrow \infty}$ norm-diam $\left(B_{i}\right)=0$.

It is known that whenever $X$ is compact and extremely disconnected, then $C(X)$ contains an isometric copy of $\ell^{\infty}$ (see [2, page 18]), therefore it is not sigma-fragmented by the norm. However, there exists a compact Hausdorff space $X$ (with the tree completeness property) such that $C(X)$ does not contain a copy of $\ell^{\infty}$ (see [4]). It is natural to ask if such a space is sigma-fragmented by the norm. The above result enable us to give an answer to this question. More precisely, thanks to Theorem 1.2, we will show that if a compact Hausdorff space $X$ with the tree-completeness property has a sequence of disjoint clopen sets, then $(C(X)$, weak) is not (sigma) fragmented by any metric which is stronger than the weak topology. It follows that $C(X)$ does not admit any equivalent locally uniformly convex norm.

2. Results. Let $T=\bigcup_{k=0}^{\infty}\{0,1\}^{k}$. The elements of $T$, are finite (possibly empty) strings of 0 's and 1's. The empty string ( ) is the unique string of length 0 ; more 
generally, the length $|t|$ of a string $t$ is $n$ if $t \in\{0,1\}^{n}$. The tree-order is defined by $s \prec t$ if $|s|<|t|$ and $t(m)=s(m)$ for $m \leq|s|$. Each $t \in T$ has exactly two immediate successors, that is, $t 0$ and $t 1$.

A topological space $X$ is said to have the tree-completeness property if whenever $\left\{V_{t}\right\}_{t \in T}$ is a sequence of disjoint clopen sets in $X$ there exists some $b \in\{0,1\}^{N^{*}}$, $N^{*}=N \cup\{0\}$, such that $\overline{\bigcup_{n \in N^{*}} V_{b \mid n}}$ is open. Evidently, every infinite extremally disconnected space [3] has the tree-completeness property. However, as it was mentioned in Section 1, there exists a compact Hausdorff space with the tree-completeness property which is not extremally disconnected.

Definition 2.1. A subset $Y$ of a compact Hausdorff space $X$ is $C^{*}$-embedded [3] in $X$ if every function in $C(Y)$ can be extended to a function in $C(X)$.

LEMMA 2.2. Let $\left\{N_{t}\right\}_{t \in T}$ be a sequence of infinite subsets of $N$, such that

(i) $N_{t} \subset N_{s}$, whenever $s \prec t$.

(ii) $N_{t} \cap N_{s}=\varnothing$, if $t$ and $s$ are not comparable.

Let $\left\{V_{n}\right\}_{n \in N^{*}}$ be a sequence of clopen subsets of a compact Hausdorff space $X$, such that $\overline{\bigcup_{k \in N_{t}} V_{k}}$ is open for each $t \in T$. If $X$ has the tree-completeness property, then there exists some $b \in\{0,1\}^{N^{*}}$, such that $\bigcup_{n=0}^{\infty}\left(X \backslash \overline{\cup_{k \in N_{b \mid n}} V_{k}}\right)$ is $C^{*}$-embedded.

Proof. Let

$$
Z_{()}=X \backslash \overline{\cup_{\left.k \in N_{(}\right)} V_{k}}, \quad Z_{t i}=\left(X \backslash \overline{\cup_{k \in N_{t i}} V_{k}}\right) \backslash \bigcup_{s \preceq t} Z_{s},
$$

for $i=0,1$ and $t \in T$. Then $\left\{Z_{t}\right\}_{t \in T}$ is a sequence of disjoint clopen subsets of $X$. By the tree-completeness property of $X$, there exists some $b \in\{0,1\}^{N^{*}}$, such that

$$
\bigcup_{n \in N^{*}} Z_{b \mid n}=\bigcup_{n \in N^{*}}\left(X \backslash \overline{\cup_{k \in N_{b \mid n}} V_{k}}\right)
$$

is clopen in $X$, thus it is $C^{*}$-embedded.

LEMMA 2.3. Let $\left\{V_{n}\right\}_{n \in N}$ be an infinite disjoint sequence of clopen subsets of a compact Hausdorff space $X$ and $\mu \in C(X)^{*}$, where $X$ has the tree-completeness property. Then there exists an infinite set $N_{1} \subset N$, such that $\overline{\cup_{n \in N_{1}} V_{n}}$ is clopen subset of $X$ and $|\mu(f)|<\epsilon$, whenever $\operatorname{supp}(f) \subset \overline{\cup_{n \in N_{1}} V_{n}}$ and $\|f\| \leq 2$.

Proof. Suppose that $2\|\mu\|<n \epsilon$. Note that for every infinite subset $M$ of $N$, there exists some infinite subset $M_{1}$ of $M$ such that $\overline{\cup_{n \in M_{1}} V_{n}}$ is clopen.

If the lemma were not true, we can find infinite disjoint subsets $M_{1}, \ldots, M_{n}$ of $N$ and continuous functions $f_{1}, \ldots, f_{n}$ such that

$$
\operatorname{supp}\left(f_{i}\right) \subset \overline{\cup_{n \in M_{i}} V_{n}} \text { (clopen), }\left\|f_{i}\right\| \leq 2, \mu\left(f_{i}\right) \geq \epsilon .
$$

Put $f=\sum_{i=1}^{n} f_{i}$, since $f_{i}$ 's have disjoint support, we have $\|f\| \leq 2$, but $\mu(f)=$ $\sum_{i=1}^{n} \mu\left(f_{i}\right) \geq n \epsilon$. This is a contradiction.

THEOREM 2.4. Let $X$ be a compact Hausdorff space with the tree-completeness property. If $X$ contains a disjoint sequence of clopen sets. Then $(C(X)$, weak) is not (sigma) fragmented by any metric which is stronger than weak topology. 
Proof. By Theorem 1.2, it is enough to show that for each strategy $\omega$ for the player $\Omega$ there exists an $\omega$-play $p=\left(A_{i}, B_{i}\right)_{i}$ such that, $\bigcap_{i \geq 1} B_{i} \neq \varnothing$ and $\lim _{i \rightarrow \infty}$ norm$\operatorname{diam}\left(B_{i}\right)>0$. Fix a strategy $\omega$ for the player $\Omega$. By induction on $|t|, t \in T$, we will construct partial $\omega$-plays $p_{t}=\left(A_{()}, B_{()}, A_{t \mid 1}, \ldots, A_{t}\right)$. Then, we will show that there is some $b \in\{0,1\}^{N^{*}}$, such that the $\omega$-play $\left.p_{b}=\left(A_{()}, B_{(}\right), A_{b \mid 1}, \ldots\right)$ has the required properties.

Let $\left\{V_{n}\right\}_{n \in N}$ be an infinite disjoint sequence of nonempty clopen subsets of $X$. Let $N_{\text {( ) }}$ be an infinite subset of $N$ such $\overline{\bigcup_{\left.n \in N_{(}\right)} V_{n}}$ is a clopen subset of $X$. For some $f_{(\text {) }}$ in the unit ball of $C(X)$, we define

$$
A_{()}=\left\{f:\|f\| \leq 1, f(x)=f_{()}(x) \text { for } x \in X \backslash \overline{\bigcup_{\left.n \in N_{(}\right)} V_{n}}\right\}
$$

as the first choice of the player $\Sigma$. Therefore, we have the partial $\omega$-play $p_{()}=\left(A_{()}\right)$, clearly norm-diam $\left(A_{()}\right)=1$. Suppose that for every $t$ with $|t| \leq n$, the partial $\omega$ play $\left.p_{t}=\left(A_{()}, B_{(}\right), A_{t \mid 1}, B_{t \mid 1}, \ldots, A_{t}\right)$ has already been defined. Let $B_{t}=\omega\left(p_{t}\right)$ be the relatively open subset of $A_{t}$, chosen by the player $\Omega$ according to his/her strategy as the answer to this movement. Let $f_{t}^{\prime} \in B_{t}$, since $B_{t}$ is a relatively open subset of $A_{t}$, there are linear functionals $\mu_{1}^{t}, \ldots, \mu_{K_{t}}^{t}$ on $C(X)$ and $\epsilon_{t}>0$, such that

$$
\left\{f \in A_{t}:\|f\| \leq 1,\left|\mu_{i}^{t}\left(f-f_{t}^{\prime}\right)\right|<\epsilon_{t}, 1 \leq i \leq K_{t}\right\} \subset B_{t} .
$$

Applying Lemma 2.3, we can find an infinite subset $N_{t}^{\prime}$ of $N_{t}$, such that $\overline{U_{n \in N_{t}^{\prime}} V_{n}}$ is clopen and

$$
\left|\mu_{i}^{t}(f)\right|<\epsilon_{t} \quad \text { whenever } \operatorname{supp}(f) \subset \overline{\bigcup_{n \in N_{t}^{\prime}} V_{n}},\|f\| \leq 2 \text { for } 1 \leq i \leq K_{t} .
$$

Suppose that $N_{t_{0}}$ and $N_{t_{1}}$ are two disjoint infinite subset of $N_{t}^{\prime}$, such that each $\overline{\bigcup_{n \in N_{t i}} V_{n}}$ is clopen, $i=0,1$. Let $f_{t i}=f_{t}^{\prime} \cdot x_{X \backslash \overline{U_{n \in N_{t i} V_{n}}}}$ and define

$$
A_{t i}=\left\{f \in A_{t}: f(x)=f_{t i}(x) \text { for } x \in X \backslash \overline{\bigcup_{n \in N_{t i}} V_{n}}\right\} \quad(i=0,1) .
$$

Then $A_{t 0}$ and $A_{t 1}$ are subsets of $B_{t}$ with norm diameter 1 and we have the partial $\omega$-plays

$$
\left.p_{t i}=\left(A_{()}, B_{(}\right), A_{t \mid 1}, B_{t \mid 1}, \ldots, A_{t}, B_{t}, A_{t i}\right) \quad(i=0,1) .
$$

Thus, by induction on $|t|$, we proved that, there are partial $\omega$-plays

$$
\left.p_{t}=\left(A_{()}, B_{(}\right), \ldots, A_{t}\right), \quad(t \in T),
$$

such that the following conditions hold:

(i) $A_{t}$ is of the form

$$
\left\{f:\|f\| \leq 1, f(x)=f_{t}(x) \text { for } x \in X \backslash \overline{\bigcup_{n \in N_{t}} V_{n}}\right\},
$$

(ii) for each $N_{t}, \overline{\bigcup_{n \in N_{t}} V_{n}}$ is clopen, 
(iii) $N_{t} \subset N_{s}$, when $s \prec t$,

(iv) $N_{t} \cap N_{s}=\varnothing$, when $s$ and $t$ are not comparable,

(v) $\operatorname{norm}-\operatorname{diam}\left(A_{t}\right)=1$ for each $t \in T$,

(vi) $f_{t}(x)=f_{t i}(x)$ for $x \in X \backslash \overline{U_{n \in N_{t}} V_{n}}$ and $i=0,1$.

Applying Lemma 2.2, we can find some $b \in\{0,1\}^{N^{*}}$, such that every continuous function on $\bigcup_{n \in N^{*}}\left(X \backslash \overline{\bigcup_{k \in N_{b \mid n}} V_{k}}\right)$ has a continuous extension on $X$. By (vi), the function $f_{b}^{*}(x)=\lim _{n \rightarrow \infty} f_{b \mid n}(x)$ is continuous on $\bigcup_{n \in N^{*}}\left(X \backslash \overline{\bigcup_{k \in N_{b \mid n}} V_{k}}\right)$, thus it has a continuous extension $f_{b}$ on $X$ without increasing norm. Clearly $f_{b} \in \bigcap_{n \in N^{*}} A_{b \mid n}$. Thus $\bigcap A_{b \mid n} \neq \varnothing$ and $\lim _{n \rightarrow \infty} \operatorname{norm}-\operatorname{diam}\left(A_{b \mid n}\right)=1$, that is, the $\omega$-play $p_{b}=\left(A_{()}, B_{()}, A_{b \mid 1}\right.$, $\left.B_{b \mid 1}, \ldots\right)$ does not satisfy Theorem 1.2(iii). This proves the theorem.

COROLLARY 2.5. If a compact Hausdorff space $X$ with the tree-completeness property has an infinite sequence of clopen sets, then $C(X)$ does not admit any equivalent locally uniformly convex norm.

Proof. It is known that if ( $C(X)$, weak) admits an equivalent locally uniformly convex norm then it is norm-fragmented (see [7, Theorem 4.2]). Thus the result follows from Theorem 2.4.

ACKNOWLEDGEMENT. The author wishes to thank the referee for his comments and careful observations.

\section{REFERENCES}

[1] J. Bourgain, $\ell^{\infty} / c_{0}$ has no equivalent strictly convex norm, Proc. Amer. Math. Soc. 78 (1980), no. 2, 225-226. MR 81b:46029. Zbl 432.46013.

[2] J. Diestel and J. J. Uhl, Jr., Vector Measures, Mathematical Surveys, no. 15, American Mathematical Society, Rhode Island, 1977. MR 56\#12216. Zbl 369.46039.

[3] L. Gillman and M. Jerison, Rings of Continuous Functions, The University Series in Higher Mathematics, D. Van Nostrand, New York, 1960. MR 22\#6994. Zbl 093.30001.

[4] R. Haydon, A nonreflexive Grothendieck space that does not contain $\ell_{\infty}$, Israel J. Math. 40 (1981), no. 1, 65-73. MR 83a:46028.

[5] R. Haydon and V. Zizler, A new space with no locally uniformly rotund renorming, Canad. Math. Bull. 32 (1989), no. 1, 122-128. MR 90g:46027. Zbl 694.46011.

[6] J. E. Jayne, I. Namioka, and C. A. Rogers, Norm fragmented weak* compact sets, Collect. Math. 41 (1990), no. 2, 133-163. MR 93d:46035. Zbl 764.46015.

[7] _ $\quad \sigma$-fragmentable Banach spaces, Mathematika 39 (1992), no. 1, 161-188. MR 93i:46027. Zbl 761.46008.

[8] _ $\quad \sigma$-fragmentable Banach spaces, Mathematika 39 (1992), no. 2, 197-215. MR 94c:46028. Zbl 761.46009.

[9] _ Fragmentability and $\sigma$-fragmentability, Fund. Math. 143 (1993), no. 3, 207-220. MR 94j:46020. Zbl 801.46011.

[10] _ Topological properties of Banach spaces, Proc. London Math. Soc. (3) 66 (1993), no. 3, 651-672. MR 94c:46041. Zbl 793.54026.

[11] J. E. Jayne and C. A. Rogers, Borel selectors for upper semicontinuous set-valued maps, Acta Math. 155 (1985), no. 1-2, 41-79. MR 87a:28011. Zbl 588.54020.

[12] P. S. Kenderov and A. K. Mirmostafaee, Nonfragmentability of Banach spaces, C. R. Acad. Bulgare Sci. 51 (1998), no. 5-6, 9-12. MR 2001f:46024.

[13] P. S. Kenderov and W. B. Moors, Game characterization of fragmentability of topological spaces, Mathematics and Education in Mathematics, 1996, Proceedings of the 25-th Spring Conference of the Union of Bulgarian Mathematicians, April 1996, Kazanlak, Bulgaria, pp. 8-18. 
[14]_, Fragmentability and sigma-fragmentability of Banach spaces, J. London Math. Soc. (2) 60 (1999), no. 1, 203-223. MR 2001f:46025. Zbl 953.46004.

[15] A. K. Mirmostafaee, On non-fragmentability of Banach spaces, Proc. Indian Acad. Sci. Math. Sci. 108 (1998), no. 2, 163-167. MR 99e:46019. Zbl 937.46012.

[16] I. Namioka, Radon-Nikodým compact spaces and fragmentability, Mathematika 34 (1987), no. 2, 258-281. MR 89i:46021. Zbl 654.46017.

[17] N. K. Ribarska, Internal characterization of fragmentable spaces, Mathematika 34 (1987), no. 2, 243-257. MR 89e:54063. Zbl 645.46017.

[18] _ A note on fragmentability of some topological spaces, C. R. Acad. Bulgare Sci. 43 (1990), no. 7, 13-15. MR 92d:54042. Zbl 763.54021.

[19] _ The dual of a Gâteaux smooth Banach space is weak star fragmentable, Proc. Amer. Math. Soc. 114 (1992), no. 4, 1003-1008. MR 92g:46020. Zbl 760.46017.

[20] J. Yu and X.-Z. Yuan, The relationship between fragmentable spaces and class $\mathscr{L}$ spaces, Proc. Amer. Math. Soc. 124 (1996), no. 11, 3357-3359. MR 97a:46016. Zbl 865.47048.

S. Alireza Kamel Mirmostafaee: Department of Mathematics, Damghan College of SCIENCES, P.O. BoX 364, DAMGHAN 36715, IRAN

E-mail address: mi rmostafa@mai 1 .com 


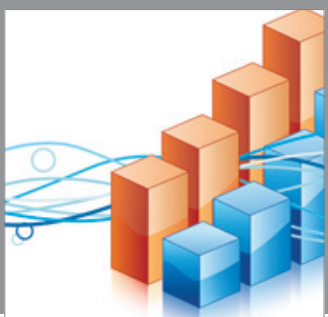

Advances in

Operations Research

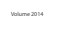

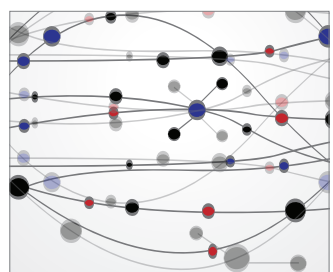

\section{The Scientific} World Journal
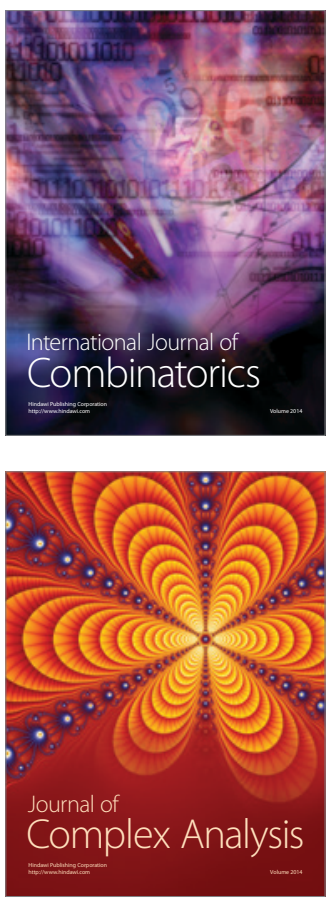

International Journal of

Mathematics and

Mathematical

Sciences
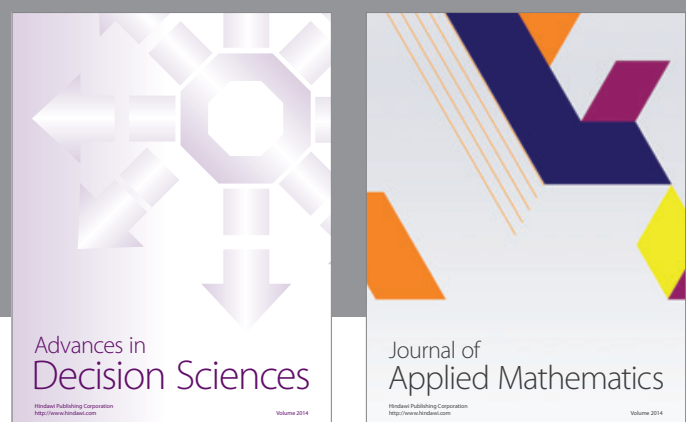

Journal of

Applied Mathematics
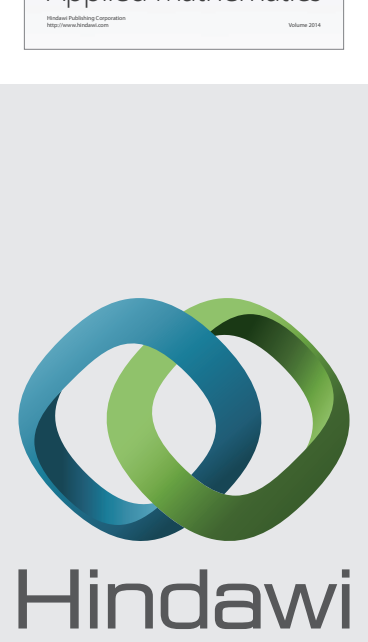

Submit your manuscripts at http://www.hindawi.com
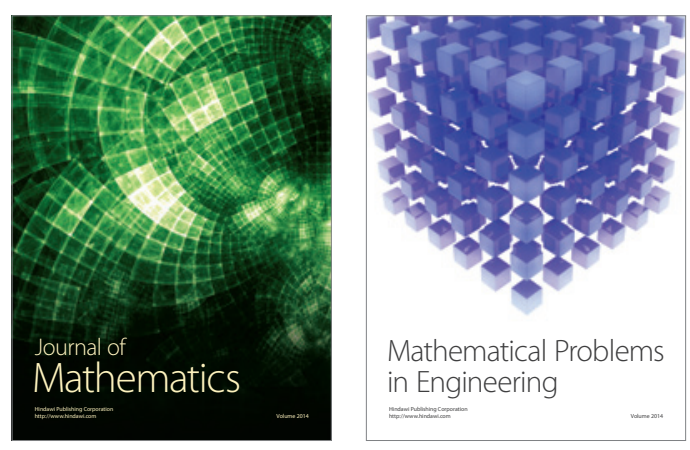

Mathematical Problems in Engineering
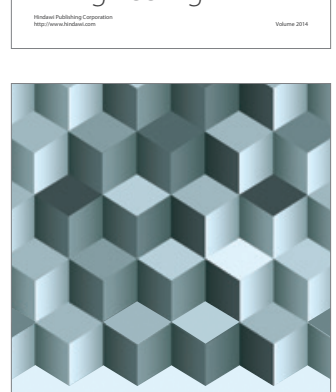

Journal of

Function Spaces
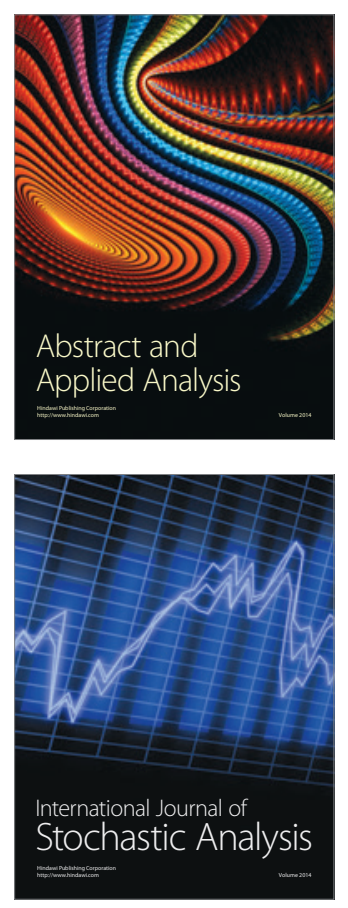

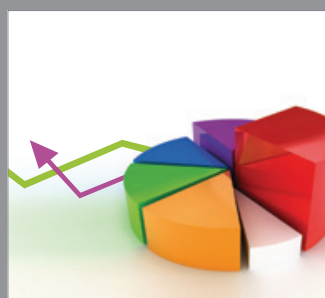

ournal of

Probability and Statistics

Promensencen
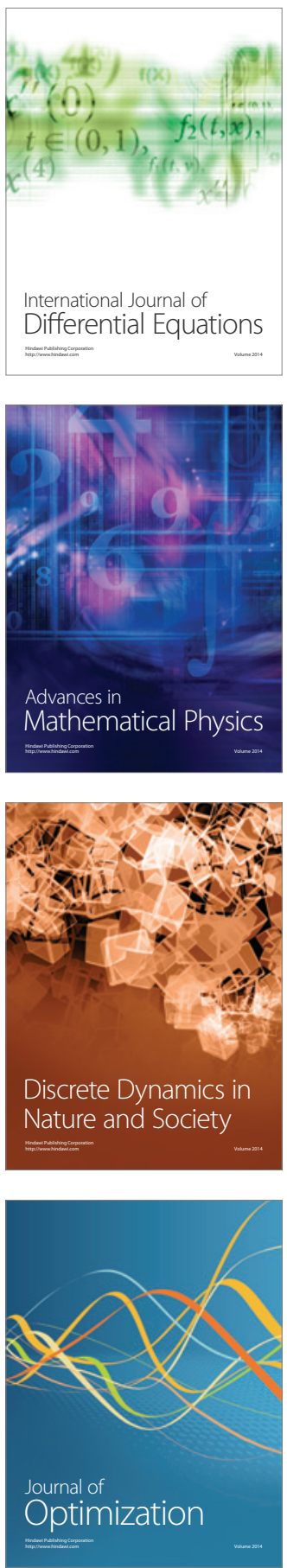